\title{
GENERIC HEAT DIFFUSION IS SCALAR CONTROLLABLE
}

\author{
LAWRENCE MARKUS
}

(Communicated by Kenneth R. Meyer)

\begin{abstract}
The partial differential equation for heat diffusion on a closed manifold $M$ is approximately controllable by a single distributed controller, under generic conditions. But we also give examples, where $M$ is a torus surface, for which no finite number of scalar controllers suffice.
\end{abstract}

Let $M$ be a smooth closed $n$-manifold ( $C^{\infty}$ with $n \geq 2$, compact without boundary). Consider the set $\mathscr{G}$ of all Riemannian metric tensor fields of class $C^{k}$ on $M$ (for any fixed $k$ on $n+4 \leq k \leq \infty$ ), so $\mathscr{G}$ with the $C^{k}$-topology has the topology of a complete metric space, and hence $\mathscr{G}$ is a Baire space; and let $\mathscr{F}=L^{2}(M, \mathbf{R})$, also topologized as a Baire space. For each pair $(g, f) \in \mathscr{G} \times \mathscr{F}$, consider the heat-diffusion control system on $M$, for the state $w(\cdot, t) \in L^{2}(M, \mathbf{R})$ at each time $t \geq 0$,

$$
\frac{\partial w}{\partial t}=\Delta_{g} w+f \cdot u(t)
$$

Here $\Delta_{g}$ is the Laplace-Beltrami operator for the metric tensor $g$, and we choose $u(t) \in L_{\text {loc }}^{1}(0, \infty ; \mathbf{R})$ as the scalar control function. We are interested in the approximate controllability of $(*)$; see [3].

We assert that the set

$$
\begin{array}{r}
C=\{(g, f) \in \mathscr{G} \times \mathscr{F} \mid(*) \text { is approximately controllable in } \\
\left.L^{2}(M, \mathbf{R}), \text { and in arbitrarily short durations }\right\}
\end{array}
$$

is residual (and hence dense) in $\mathscr{G} \times \mathscr{F}$. We paraphrase this assertion in the

Theorem. Generic heat diffusion is scalar controllable.

Proof. Define $\mathscr{G}_{s}=\left\{g \in \mathscr{G} \mid \Delta_{g}\right.$ has only simple eigenvalues $\}$, and note that $\mathscr{G}_{s}$ is residual in $\mathscr{G}$; see [4]. Now consider the set $Z=\left\{(g, f) \in \mathscr{G} \times \mathscr{F} \mid g \in \mathscr{G}_{\text {s }}\right.$ and $f$ has all nonzero Fourier coefficients for the eigenbasis of $\left.\Delta_{g}\right\}$.

Suppose that $Z$ is of first category in the Baire space $\mathscr{G} \times \mathscr{F}$. In this case [2, $\S 22 \mathrm{~V}]$, there exists some $g_{0} \in \mathscr{G}_{s}$ such that $Z \cap \mathscr{F} g_{0}$ is of first category in the "vertical section" $\mathscr{F} g_{0}=\left(g_{0}\right) \times \mathscr{F} \subset \mathscr{G} \times \mathscr{F}$. This is a contradiction since the set $\{f \in \mathscr{F} \mid f$ has all nonzero Fourier coefficients for a prescribed orthonormal

Received by the editors June 12, 1990.

1980 Mathematics Subject Classification (1985 Revision). Primary 58G11; Secondary 93C20.

Research done under NSF Grant DMS 87-22402. 
basis in $\left.L^{2}(M, \mathbf{R})\right\}$ is clearly residual in $\mathscr{F}$. Hence $Z$ must be residual. But $C \supset Z$, see $[1,3]$, so $C$ is also residual in $\mathscr{G} \times \mathscr{F}$.

Similar results hold for the case where $M$ is a region in some ambient manifold, where $\bar{M}$ is compact and Dirichlet conditions apply on the smooth boundary $\partial M$-and, for more general linear parabolic systems.

Example. Consider the heat-diffusion distributed parameter control system

$$
\frac{\partial w}{\partial t}=\frac{\partial^{2} w}{\partial x^{2}}+\frac{\partial^{2} w}{\partial y^{2}}+f_{1}(x, y) u_{1}(t)+\cdots+f_{m}(x, y) u_{m}(t) \quad \text { for } t \geq 0
$$

on a locally flat torus surface $T^{2}$ defined by the translations of the Euclidean $(x, y)$-plane

$$
\begin{array}{ll}
x \rightarrow x+2 \pi \alpha, & x \rightarrow x, \\
y \rightarrow y, & y \rightarrow y+2 \pi \beta,
\end{array} \quad \text { fixed real } \alpha>0, \beta>0 .
$$

Here the $m$ functions $f_{j}(x, y) \in L^{2}\left(T^{\cdot 2}\right)$ and the scalar controllers $u_{j}(t) \in$ $L_{\text {loc }}^{1}(0, \infty ; \mathbf{R})$ (for $j=1, \ldots, m$ ) are as before. A necessary condition for the approximate controllability of this system is that each eigenspace $E_{\lambda}$ of the Laplacian has a dimension $\leq m$; see [3]. We assume that $\alpha / \beta=p / q$ is a rational number (here $p, q$ are positive integers), and show that $\sup \operatorname{dim} E_{\lambda}=$ $\infty$.

Take positive integers $a_{1}, b_{1}$ so that the number $\left(a_{1}\right)^{2}+\left(b_{1}\right)^{2}$ can be written as the sum of two integral squares in more than $m$ distinct ways. Define $a=a_{1} p, b=b_{1} q$, and compute

$$
\frac{a^{2}}{\alpha^{2}}+\frac{b^{2}}{\beta^{2}}=\frac{p^{2}}{\alpha^{2}}\left[\left(a_{1}\right)^{2}+\left(b_{1}\right)^{2}\right] .
$$

But $\phi(x, y)=\sin (a x / \alpha) \sin (b y / \beta)$ is an eigenfunction for the Laplacian, with eigenvalue $\lambda_{a b}=-\left[a^{2} / \alpha^{2}+b^{2} / \beta^{2}\right]=-\left(p^{2} / \alpha^{2}\right)\left[a_{1}^{2}+b_{1}^{2}\right]$. Thus, for each pair of integers $\hat{a}, \hat{b}$ with $\hat{a}^{2}+\hat{b}^{2}=a_{1}^{2}+b_{1}^{2}$, we have a corresponding pair $\hat{a} p, \hat{b} q$ defining an eigenfunction $\hat{\phi}(x, y)=\sin (\hat{a} p x / \alpha) \sin (\hat{b} q y / \beta)$ with the same eigenvalue $\lambda_{a b}=-\left(p^{2} / \alpha^{2}\right)\left[\hat{a}^{2}+\hat{b}^{2}\right]$. Therefore $\operatorname{dim} E_{\lambda_{a b}}>m$, and clearly $\sup \operatorname{dim} E_{\lambda}=\infty$.

On each smooth closed $n$-manifold $M$ define the subset of Riemann metrics

$$
\mathscr{G}_{r}=\left\{g \in \mathscr{G} \mid \text { eigenspaces } E_{\lambda} \text { of } \Delta_{g} \text { satisfy } \sup \operatorname{dim} E_{\lambda}=\infty\right\} \text {. }
$$

It would be interesting to know under what circumstances $\mathscr{G}_{r}$ is dense in $\mathscr{G}$.

\section{REFERENCES}

1. H. O. Fattorini, On complete controllability of liner systems, J. Differential Equations 3 (1967), 391-402.

2. K. Kuratowski, Topology, vol. 1, Academic Press, New York, 1966.

3. L. Markus, Introduction to the theory of distributed control systems, Distributed Parameter Control Systems: Trends and Application Systems, Marcel Dekker, NY, 1990.

4. K. Uhlenbeck, Generic properties of eigenfunctions, Amer. J. Math. 98 (1976), 1059-1078. 